NASA Technical Memorandum 106330

$$
\begin{gathered}
1 N 35 \\
186038 \\
19 P
\end{gathered}
$$

\title{
Tomographic Methods in Flow Diagnostics
}

Arthur J. Decker

Lewis Research Center

Cleveland, Ohio

Prepared for the

Summer School on Optical Diagnostics for Flow Processes cosponsored by The Danish Research Academy and the European Community Programme on Human Capital and Mobility Roskilde, Denmark, September 26-October 2, 1993 


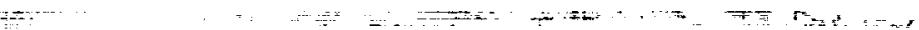

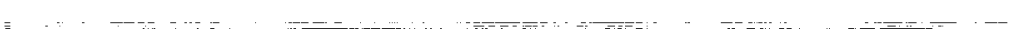




\title{
TOMOGRAPHIC METHODS IN FLOW DIAGNOSTICS
}

\author{
Arthur J. Decker \\ National Aeronautics and Space Administration \\ Lewis Research Center \\ Cleveland, Ohio 44135
}

\section{SUMMARY}

This report presents a viewpoint of tomography that should be well adapted to currently available optical measurement technology as well as the needs of computational and experimental fluid dynamists. The goals in mind are to record data with the fastest optical array sensors; process the data with the fastest parallel processing technology available for small computers; and generate results for both experimental and theoretical data. An in-depth example treats interferometric data as it might be recorded in an aeronautics test facility, but the results are applicable whenever fluid properties are to be measured or applied from projections of those properties. The paper discusses both computed and neural net calibration tomography. The report also contains an overview of key definitions and computational methods, key references, computational problems such as ill-posedness, artifacts, and missing data, and some possible and current research topics.

\section{INTRODUCTION}

Flow visualization often displays the integral transforms of flow properties rather than the properties themselves. Interferometry is an example. A double-exposure hologram of two index-of-refraction fields $n_{0}(x, y, z)$ and $n(x, y, z)$, recorded, for example, from light propagating parallel to the $z$-axis, yields, in the refractionless limit, interference phase measurements given by

$$
\Delta \phi(x, y)=\frac{2 \pi}{\lambda} \int\left(\mathrm{n}(\mathrm{x}, \mathrm{y}, \mathrm{z})-\mathrm{n}_{0}(\mathrm{x}, \mathrm{y}, \mathrm{z})\right) \mathrm{d} \mathrm{z}
$$

One measurement of the interference phase given by (1), at one point $x, y$, represents one sample point of the so-called $x$-ray transform of $n(x, y, z)-n_{0}(x, y, z)$. The calculation of $n(x, y, z)-n_{0}(x, y, z)$ from such sample points is an example of computed tomography. High speed detector arrays, powerful small computers, fast parallel processors, and a body of research conducted in the 1970's and particularly in the 1980's make tomography a viable tool to be considered for flow diagnostics.

There are at least three integral transforms of interest to tomographers: the Radon transform, the fan-beam or cone-beam transform, and the x-ray transform. We shall consider only the Radon and x-ray transforms and shall note in particular how the two transforms differ.

The Radon transform, first described by Johann Radon in 1917 (ref. 1), is defined for any number of dimensions $\mathrm{n}$ in Euclidean space $\mathbf{R}^{\mathrm{n}}$. The Radon transform $\mathrm{R} f(x)$ of a function $\mathrm{f}(\mathrm{x})$ where $\mathrm{x}=\left(\mathrm{x}_{1}, \mathrm{x}_{2}\right.$, $\left.\ldots, x_{n}\right) \in \mathbf{R}^{n}$ is the set of all integrals of $f(x)$ over all the hyperplanes of $\mathbf{R}^{n}$.

The x-ray transform, by contrast, is always a straight-line integral. It is also defined for any number of dimensions n. Consider all planes through the origin. A plane through the origin is defined by the coordinates $\theta$ of its normal. Consider a particular plane through the origin and any straight line perpen- 
dicular to that plane. The $x$-ray transform $P f(x)$ of a function $f(x)$ is the set of all integrals along all straight lines perpendicular to all planes through the origin. In fact, the definition of the $x$-ray transform is unaffected by the location of the plane perpendicular to $\theta$. One can imagine a plane in three dimensions being translated along $\theta$ and perpendicular to $\theta$ until it coincides with an interferogram of a fluid. The assumption is that the entire fluid projects onto the interferogram in this manner. The continuum of interference phase measurements (except a possible reference offset and normalization) is the x-ray transform at $\theta$ of the index of refraction of the fluid. Defining the complete $x$-ray transform requires recording and measuring interferograms for the continuum of directions on the unit hemisphere.

The $\mathrm{x}$-ray and Radon transforms are the same in two dimensions. Medical tomography using x-rays is done typically by stacking two-dimensional slices recorded through a patient. It's common and correct to refer to the linear projections recorded for a slice as samples of the Radon transform. The terminology may mislead flow diagnostics professionals who may need to use three-dimensional tomography at times. Figure 1 summarizes the difference between the Radon and $x$-ray transforms in $\mathbf{R}^{3}$. An integral of a function over the plane shown in the figure is a sample point of the Radon transform, and an integral along the line perpendicular to the plane is a sample point of the $\mathrm{x}$-ray transform.

Computed tomography consists of estimating a function or property from measured or theoretical samples of the integral transforms. (Theoretical objects and their transforms are called phantoms.) Figure 2 shows a division of tomography into three major efforts: fast measurements of the transforms; reliable, accurate algorithms or processors for inverting the transforms; and utilization of the computations. In medical tomography, each effort has its own dedicated experts. Tomography is a legitimate branch of applied mathematics and has its own full-time practitioners. The methods employed depend strongly on the measurement technology and the end uses. Tomography is underdeveloped for flow diagnostics. Because this is only a brief overview, it's important to mention some good references.

Perhaps the best general reference is F. Natterer's The Mathematics of Computerized Tomography (ref. 2). This book contains excellent discussions and examples of the effects of errors due to sampling, illposedness, and missing data. The book also discusses the principal algorithms for inverting the transforms. This reference was written by a mathematician for mathematicians, and the terminology may be difficult for other readers.

S.R. Dean's The Radon Transform and Some of its Applications (ref. 3) discusses the principal algorithms in notation that are probably more familiar to engineers. The book has an extensive list of references. Unfortunately, there are no practical discussions of the effects of errors, and such discussions are indispensable for the application of computed tomography to flow diagnostics, especially for the incomplete data cases. Dean's and Natterer's books are excellent as a pair.

The book, Solution of Ill-posed Problems, by A.N. Tikhonov and V.Y. Arsenin (ref. 4) is not a tomography book, but it does exp节 the origin and control of ill-posedness (the amplification of errors depending on their spectral content).

The computational methods that accompany the algorithms of tomography are discussed practically and understandably by W.H. Press, et al. in Numerical Recipes (ref. 5).

Tomography has its own journals, although they don't normally discuss flow diagnostics. The Journal of Computer Assisted Tomography (ref. 6) discusses medical applications; Inverse Problems (ref. 7) contains papers by applied mathematicians and physicists. 
The remainder of this overview will discuss: measurements of the integral transforms for flow diagnostics; algorithms for inverting the x-ray transform; three-dimensional tomography using incomplete data from interferograms as an example; computers and artificial neural networks for tomography; and some on-going research.

\section{SAMPLING THE INTEGRAL TRANSFORMS FOR FLOW DIAGNOSTICS}

Useful tomography requires the parallel, or at least fast, measurement of thousands of channels of data. Some optical technologies such as phase shifting interferometry with CCD cameras meet this requirement. The next section discusses sampling the Radon transformation in three dimensions.

\subsection{Sampling the Radon Transformation in Three Dimensions}

It is mathematically expedient to assume that the fluid property is non-zero only within a sphere. The theorems of tomography assume that a function has this so-called compact support. It's convenient to normalize coordinates so that the fluid is confined to within a spherical region of unit radius called the unit ball $\Omega^{3}$, and the property itself is often normalized. These stipulations are kept in mind, if not stated explicitly, in the following discussions.

Hanson, et al. (ref. 8) have discussed at least the concept of measuring various fluid properties from laser-induced fluorescence produced by sheet or planar illumination. Consider the case where effects other than density can be ignored. Imagine firing a pulsed laser sheet. The total light emitted in fluorescence is proportional to the number of fluorescing molecules $N(\theta, r)$ in a thin sheet located about the plane defined by radial coordinate $r$ and orientation $\theta$. We could imagine surrounding the region with a spherical calorimeter with a thin slice through which the laser sheet passes unincumbered. The total energy measured is essentially one sample of the three-dimensional Radon transform of the density $\rho(x)$. In a sense, the number of fluorescing molecules is a sample of the Radon transform of the density in the limit where the sheet has zero thickness. In mathematical terms the energy measured is given by

$$
\mathrm{E}(\boldsymbol{\theta}, \mathrm{r})=\tau \mathrm{N}(\boldsymbol{\theta}, \mathrm{r})=\tau \mathrm{t} \int_{\mathbf{x} \cdot \theta} \rho \mathbf{r}(\mathbf{x}) \mathrm{d} \mathbf{x}
$$

where $t$ is the thickness of the sheet and $\tau$ is a proportionality factor.

This concept is extended as shown in figure 3. The laser beam is split into multiple sheets. The sheets are separated by large enough distances that times of flight within sheets can be neglected in comparison with the times of flight between sheets. A fast point detector then reads out the samples of the Radon transformation as a string of pulses. Each value of $\theta$ must be sampled in the same manner, for example, by routing subsequent laser pulses into different paths, where each path has a point detector.

The fluorescence source function might depend on several thermodynamic variables, but the exercise can be performed at several wavelengths. One might even be tempted to extend the exercise to velocity, but here the concept fails. The propagation direction of the beam and component of velocity measured change for each sample direction. The tomography literature generally assumes a scalar field; vector-field tomography is a topic for research.

We will say very little else about three-dimensional Radon transformations. Should you have an appropriate application or practical measurement method, Natterer (ref. 2) provides an adequate discus- 
sion. The Radon transform is probably mathematically simpler than the $\mathrm{x}$-ray transform and exhibits a certain locality. This locality is shown by the inverse of (2), which is given by the equation

$$
\rho(x)=-\left.\frac{1}{8 \pi^{2} t} \int_{S^{2}} \frac{\partial^{2}}{\partial p^{2}} N(\theta, p)\right|_{p}=x \cdot \theta=r^{d} \theta
$$

The density at $\mathbf{x}$ is recovered effectively from (all) planes in the neighborhood of $\mathbf{x}$. The summation over $\boldsymbol{\theta}$, after the differentiation, is an example of back projection.

The Radon transform is not as robust as the x-ray transform for work in three dimensions, particularly if data are limited. The Radon transform involves only three degrees of freedom (two angular and one positional), while the x-ray transform offers four degrees of freedom (two angular and two positional). The Radon transform is more sensitive to data errors (round-off or measurement). For relative error $\varepsilon$, the Radon transform deteriorates as $\varepsilon^{1 / 3}$, and the X-ray transform deteriorates as $\varepsilon^{1 / 2}$ (ref. 9).

\subsection{Sampling the X-ray Transform in Two and Three Dimensions}

Visualization data is recorded effectively as an x-ray transform. Figure 4 shows the fluid depicted in figure 3, but with the entire volume illuminated. An afocal imaging system produces approximately a parallel projection of the illuminated volume. An array detector samples the x-ray transform for the direction shown. Array detectors would be required for each direction. Even velocity could be measured in principle for this concept; since the illumination beam has a fixed direction.

Diffuse-illumination holographic interferometry is the classical method for generating the $\mathrm{x}$-ray transform in three dimensions. Phase shifting or heterodyne detection can be used for efficient interrogation of the hologram. Later we discuss an example using heterodyne detection. The interference phase, measured in interferometry of fluids in the refractionless limit, is proportional to the x-ray transform of index of refraction as mentioned in the introduction.

Deflectometry (schlieren, moire, Hartmann screens) projects components of the gradient of the index of refraction of a fluid. Deflectometry offers high speed electronic array detection. However, the vector compositions of the components measured vary with direction $\theta$, thereby preventing deflectometry from being used with standard tomographic methods.

The $x$-ray transform of a fluid property $f(x)$ is given, in general, by the equation

$$
P_{\theta} f(x)=\int_{-\infty}^{\infty} f(x+t \theta) d t
$$

where $\theta$ is the direction in two or three (or even $\mathrm{n}$ ) dimensions, and $\mathbf{x}$ here is the coordinate of a point in the projection plane. Naive attempts to invert (4), even from good measurements, may lead to poor results unless correct algorithms are selected and applied correctly.

\section{ALGORITHMS FOR INVERTING THE X-RAY TRANSFORM}

S.H. Izen has studied the $\mathrm{x}$-ray transform extensively for $\mathrm{n}$ dimensions for both full and missing data (refs. 10 to 13). The work is discussed in a form suitable for applications to flow diagnostics by A.J. Decker and S.H. Izen (ref. 14). Prior to this work, most generalizations of computed tomography to 
three or more dimensions were applied to the Radon transform. The methods for inverting the $x$-ray and Radon transforms in two dimensions are, of course, identical.

The two major sources of poor performance are undersampling or discretization and ill-posedness. Systematic errors such as those caused by misregistration of projections also can be serious. The consequences of errors appear as incorrect reconstructions called artifacts (artefacts). Incomplete data due to view limitations or obstructing objects worsens these effects. (By complete data, we mean a uniform sampling of the entire x-ray transform according to the Nyquist criterion.) Natterer (ref. 2) shows prints of the effects of sampling, ill-posedness, and incomplete data. The origins of these effects can be understood in terms of the projection slice theorem.

\subsection{Projection Slice Theorem}

The projection slice theorem states that the n dimensional Fourier transform (or inverse transform) of a property in any plane through the origin of the transform space equals the $n-1$ dimensional Fourier transform (or inverse transform) of the $\mathrm{x}$-ray transform in a parallel plane. The inverse Fourier transform of $f(x)$ is defined as

$$
F_{n}^{-1} f(x)=(2 \pi)^{-n} \int_{R^{n}} \exp (i x \cdot k) f(x) d x
$$

Inverse Fourier transforms simplify the notation for the in-depth example to be presented later.

The projection slice theorem in three dimensions is then given by the equation

$$
(2 \pi) \mathrm{F}_{3}^{-1} \mathrm{f}(\eta)=\left[\mathrm{F}_{2}^{-1} \mathrm{P}_{\theta} \mathrm{f}\right](\eta)
$$

where $\eta$ represents Fourier transform coordinates on planes perpendicular to $\theta$. The left member of (6) refers to a slice through the three-dimensional object, and the right member refers to the $x$-ray transform plane.

The direct use of (6) (or its two-dimensional version) in tomography is called a Fourier reconstruction technique. The projection slice theorem produces some immediate insights.

The first insight is that the sampling requirements for the object and the x-ray transform are the same. If $\mathbf{k}$ for the property distribution is confined essentially to a sphere of radius $b$, then the distance $h$ between samples of the $\mathrm{x}$-ray transform should satisfy

$$
\mathbf{h} \leq \frac{\pi}{\mathrm{b}}
$$

(A function with compact support is only essentially band limited.)

The second insight is that missing data impose no fundamental restrictions on the inversion of the $\mathrm{x}$-ray transform. The Fourier transform of a function with compact support is analytic. The entire transform can be recovered by (error-free) analytic continuation starting from its (error-free) representation in 
any region. Restrictions are, in fact, imposed by the computational method and by ill-posedness. Analytic continuation is ill-posed as are all procedures for inverting the $\mathrm{x}$-ray transform.

\subsection{Ill-Posedness and Regularization}

Ill-posedness (refs. 4 and 14) can be thought of as the nonuniform response of a computational procedure to the modal content (in any representation) of the errors. Errors appear in measurements, machine precision, or round-off. Ordinary differentiation is ill-posed, for example. If the error contains modes $\sin (\mathrm{ky})$ and $\cos (\mathrm{ky})$, then the derivative contains corresponding errors that vary as kcos(ky) and $-\mathrm{ksin}(\mathrm{ky})$. Anyone who has attempted to differentiate noisy data has seen the effect. The second derivative in (3) for inverting the Radon transform clearly imparts larger effects to errors in the higher frequency components.

The general filtering procedures used to control ill-posedness are called regularization. Regularization is defined in terms of a regularization parameter $\gamma(\varepsilon)$ for error $\varepsilon$, where $\gamma \Rightarrow 0$ as $\varepsilon \Rightarrow 0$. The regularization parameter's form and application depend on the computational procedure for inverting the $\mathrm{x}$-ray transform. Three procedures are discussed briefly: the algebraic reconstruction technique (ART), convolution back projection, and series methods (considered examples of the Fourier reconstruction technique).

\subsection{Algebraic Reconstruction Technique (ART)}

An algebraic reconstruction technique (ART) can be defined to be any iterative technique for inverting the Radon or $\mathrm{x}$-ray transforms (ref. 15). The usual procedure is to discretize the property distribution. For the two-dimensional case, the property value can be assumed to be constant in each finite object element. The object elements are called pixels since the results of the reconstruction are often displayed electronically. Each sample of the x-ray transform then involves only a few pixels (about $\mathbf{M}^{1 / 2}$ where $M$ is the number of pixels). The contribution of pixel $m$ to the $j$ value $g_{j}$ of the $x$-ray transform is $a_{j m} f_{m}$ where the coefficient $a_{j m}$ can be equated to the length of the projection line segment that intersects the pixel (fig. 5). A sample point of the $x$-ray transform is then given by

$$
g_{j}=\sum_{m=1}^{M} a_{j m} f_{m}
$$

where $g_{\mathrm{j}}$ is measured in the manner described in section 2.2 , and the $a_{j m}$ are calculated from geometry. The various sample points of the x-ray transform are then used to build a set of linear equations. Most of the coefficients vanish, thereby yielding a sparse matrix equation that is well suited, in principle, for solution by iterations.

One procedure (ref. 16) follows. Start with a guess $F_{0}$ of the $M$ pixel averaged values of the property $f$. Let $a_{j}$ be the vector of coefficients for sample point $j$ of the x-ray transform. Execute in order the following $\mathrm{N}$ computations for the $\mathrm{N}$ measured sample points of the $\mathrm{x}$-ray transform:

$$
F_{j}=F_{j-1}+\frac{\omega}{\left|a_{j}\right|^{2}}\left(g_{j}-a_{j}^{T} F_{j-1}\right) a_{j}
$$


The parameter $\omega$ is called a relaxation parameter and is selected in the interval $(0,2)$. Each pass through the $\mathbf{N}$ computations is called one iteration.

Generally, there is an optimum number of iterations. The reconstruction appears to improve until that number is reached, and then artifacts increase with more iterations and destroy the reconstruction. This phenomenon is the manifestation of ill-posedness in ART. The reciprocal of the optimum number of iterations can be thought of as the regularization parameter $\left(\gamma(\varepsilon) \sim 1 / \mathrm{N}_{\text {OPTIMUM }}(\varepsilon)\right)$.

Although ART does not seem to be regarded as a good technique, that judgement is often made while comparing ART with back projection. C.M. Vest discusses the use of the above approach to ART with holographic interferometry (ref. 17).

\subsection{Convolution Backprojection}

Convolution backprojection is the signature method for performing medical $\mathrm{x}$-ray computed tomography. It is very difficult to meet the requirements of the method in flow diagnostics. Backprojection, by definition, requires projections from all viewing directions. The technique is applied to two-dimensional slices, and the slices are stacked for complete information. Each slice may require a large number of sample points $(50,000$ to 200,000$)$. Generally, there should be more views than sample points per view. R.M. Lewitt presents a good overview of the technique for the x-ray and fan beam transforms (ref. 18).

A regularized integral form of the algorithm is summarized by the following equations. The coordinates within a slice are denoted by $(x, y)$. The first equation is the backprojection operation itself.

$$
f(x, y)=\int_{0}^{\pi} p^{c}(x \cos \theta+y \sin \theta, \theta) d \theta
$$

Here, $\mathrm{p}^{\mathrm{c}}$ is the projection after convolution given by the equation

$$
\mathrm{p}^{\mathrm{c}}(\hat{\mathrm{s}}, \theta)=\int_{-1}^{1} \mathrm{p}(\mathrm{s}, \theta) \mathrm{q}(\hat{\mathrm{s}}-\mathrm{s}) \mathrm{ds}
$$

where $\mathrm{p}(\mathrm{s}, \theta)$ is a sample point of the $\mathrm{x}$-ray transform at distance $\mathrm{s}$ from the origin on a line at angle $\theta$ with respect to the $x$-axis. The function $f(x, y)$ is assumed to have compact support on $\Omega^{2}$.

The convolving function, including a band limiting window, is given by the equation

$$
q(s)=(2 \pi)^{-2} \int_{-\pi / \Delta s}^{\pi / \Delta s}|k| W(k) \exp (i k s) d k
$$

Equations (10) to (12) must be discretized and an appropriate window W(k) supplied for band limiting and regularization (ref. 18).

Two-dimensional backprojection techniques are preferred, if there are no limits on views. One application to flow diagnostics is for external rotating flows as might be associated with a rotating helicopter blade. Multiple holograms can be recorded, for example, at a fixed station as the flow rotates by. 


\subsection{Series Method}

A series method is simply an application of the Fourier reconstruction technique to a series representation of a property. Series methods have very useful properties for flow diagnostics. In effect, projection data, model data, and other measurements of a property can be combined easily with a series method.

The simplest approach is to represent the property distribution in terms of orthonormal basis functions $\mathrm{V}_{\mathbf{n}}(\mathbf{x})$ as in

$$
f(x)=\sum_{\mathbf{n}} A_{\mathbf{n}} V_{\mathbf{n}}(\mathbf{x})
$$

where $\mathbf{n}$ represents the indices of the basis functions. The number of indices equals the number of dimensions.

Equation (13) is transformed a term at a time to yield

$$
F^{-1} f(\eta)=\sum_{n} A_{n} F^{-1} V_{n}(\eta)
$$

where $\eta$ represents the coordinates in inverse Fourier space. Each $F^{-1} V_{n}(\eta)$ can be evaluated analytically. But, by the projection slice theorem (6), each Fourier transformed $x$-ray sample point has its own Fourier coordinates $\eta_{j}$, and can be equated, except factors of $2 \pi$ to (14) at the corresponding coordinate.

The computational procedure is to terminate (14) and write an equation in the remaining coefficients $A_{n}$ for each transformed sample point. The orthonormal functions, in general, will oscillate, and the series is terminated for functions that oscillate too rapidly for the sampling rate (speaking approximately). There are usually many more sample points than coefficients so that a large, overdetermined set of equations is obtained. This set is expressed by the matrix equation

$$
\mathrm{Q} \mathbf{a}=\mathbf{b}
$$

where $\mathbf{a}$ is the vector of unknown coefficients, $\mathbf{b}$ is the generally much larger vector of Fourier transformed sample points of the x-ray transform, and $Q$ is a matrix computed from the transformed orthonormal functions.

A second regularization step (in addition to termination of the series) is required in this case. Computing the generalized inverse of $Q$ is an ill-posed problem that may be severely ill-posed for missing data. Singular value decomposition (SVD) is used to accomplish both inversion and regularization. For SVD, Q is written as

$$
\mathrm{Q}=\mathrm{UWV} \mathrm{V}^{\mathrm{T}}
$$

where $\mathrm{U}$ and $\mathrm{V}$ are column orthogonal matrices, and $\mathrm{W}$ is diagonal, containing the so-called singular values. In effect, computing the SVD solves the problem, since a can be estimated from $\mathrm{V} \mathrm{W}^{-1} \mathrm{U}^{\mathrm{T}} \mathrm{b}$. In fact, doing so may lead to significant errors, if the data contains measurement errors. The so-called condition of $Q$ is measured by the ratio of the largest singular value to the smallest singular value. $A$ large ratio means that $Q$ is ill-conditioned for computing a generalized inverse. Regularization consists of zero- 
ing the singular values which differ by a large factor from the maximum singular value. The effect of zeroing a singular value is to remove the corresponding column vectors in $\mathrm{U}$ and $\mathrm{V}$ from the calculation. The penalty is the loss of information associated with those components. The benefit is the removal of an error amplification factor proportional to the reciprocal of the small singular value. The reciprocal of the retained singular value ratio should be approximately equal to the error $\varepsilon$.

The series method is used the same way for complete and incomplete data. Equation (13) can also be used to incorporate presumed known values of a property. These known values are called constraints. Constraints might be known from other measurements or they might be inserted from a model. Each known value of $f(x)$ produces from (13) one additional equation in the coefficients. These equations are simply appended to the set used to form (15).

The series method does have some significant disadvantages. The orthonormal functions are nonlocal, and errors in computing a coefficient appear throughout the volume. There is a so-called Gibbs phenomenon, which makes it hard to recover edges such as those occurring at shock waves. The use of local functions called wavelets are being investigated to alleviate these problems. A specific example of the use of the series method is presented next.

\section{THREE-DIMENSIONAL TOMOGRAPHY USING INTERFEROGRAMS}

The series method was used to evaluate the efficacy of computed tomography for the limited angle problem. The limited angle problem, as shown in figure 6 , refers to a cone of viewing directions with the cone angle less than 90 degrees. The objective of this project was to evaluate computed tomography for very small cone angles of about 10 degrees. This angle limitation is representative of internal flow diagnostics (the flow diagnostics within the components of a jet engine). A single diffuse-illumination hologram will record projections within a cone angle of about 10 degrees (20 degrees between extreme views).

The evolution of this project is discussed in several references (refs. 10 to 14). The detail in those references is beyond the scope of this overview. The objective here is to outline how tomography can be analyzed for a potential application to flow diagnostics.

One decision was to use three-dimensional computed tomography to combat the extreme illposedness. Two-dimensional tomography ignores slice-to-slice projections and thereby discards data that can be measured naturally in flow diagnostics. Nevertheless, two-dimensional tomography is computationally much easier. Convolution backprojection produces the best results for full data.

Another decision was to use a standard test object or phantom. The phantom was a ball having a constant index of refraction and unit diameter. The ball was contained in the unit-radius space. The region outside the ball, of course, had a different index of refraction. This phantom represents a spherical shock wave. It is important to note that there were no a priori assumptions about the symmetry of the phantom. Convex polyhedra were also used as phantoms.

A final decision was to assume the use of infinite fringe interferograms measured to an accuracy of $1 / 50$ fringe. This conservative choice probably means that singular values that differ from the maximum by more than a factor of 50 should be rejected.

Interferograms were computed for the phantom to serve as inputs to the tomography routine. The original study used 29 interferograms, where 28 interferograms were arranged in nearest neighbor fashion 
about a central interferogram. Each interferogram contained $32 \times 32$ samples of the computed interference phase. Hence, there were 29,696 computed sample points.

The orthonormal functions $V_{n}(x)$ of (13) were products of spherical harmonics and Jacobi polynomials, and the inverse Fourier transformed functions $F^{-1} V_{n}(\eta)$ of $(14)$ were products of Bessel functions of the first kind and spherical harmonics. There is a software package, created during this project, that performs the singular value decomposition for any cone angle, calculates phantoms with and without noise, handles data from real interferograms, and can add constraints (ref. 19).

The termination of the series in (13) depends on the overall maximum degree of the polynomials to be retained. The polynomials are composed of Jacobi and associated Legendre contributions. The number of coefficients is given by

$$
N(\mathrm{~S})=\frac{(\mathrm{S}+1)(\mathrm{S}+2)(\mathrm{S}+3)}{6}
$$

where $S$ is the maximum degree of the polynomials retained. The referenced study (ref. 14) was performed for $S=12$ and $N=455$. Here, 29,696 equations are solved for 455 coefficients. Required computer resources will be discussed briefly in the next section.

The figures 7 to 9 show the results for a lesser calculation, which was performed for tutorial purposes. The calculation was performed for $2916 \times 16$ samples of the x-ray transform for a total of 7424 samples. The series was terminated at degree $S=8$ for $N=165$ coefficients.

Figure 7 shows the relative singular-value spectra (diagonal elements of $W$ ) for three geometries. Remember that calculating the regularized generalized inverse of $Q$ in (15) depends on the cone angle and accuracy, but not on the phantom. The geometries consist of a 10-degree viewing cone, a pair of 10-degree viewing cones at right angles, and a 90-degree viewing cone. Ill-posedness is seen to range from mild for the 90-degree (full view) case to extreme for the 10-degree case.

Only 105 singular values are retained for the single 10-degree cone; whereas 163 singular values are retained for the pair of 10 -degree cones. The full view easily retains all 165 singular values.

Figure 8 shows the density values computed from the phantom interferograms. The density values were computed on a $16 \times 16 \times 16$ grid from the coefficients and orthonormal functions. The single viewing cones are defined to be along the z-axis, and only 16 values along a line parallel to that axis are plotted. In other words, the z-axis is perpendicular to the viewing window, and figure 8 shows the performance of tomography along this hard-to-recover direction.

We conclude that tomography with a 10-degree view limitation is not able to measure enough singular vectors even at $S=8$ and cannot recover the edge of the sphere adequately. The 90-degree or full-view case is able to make full use of $S=8$ and would easily benefit from higher resolution interferograms and higher order polynomials. The pair of cones makes full use of $S=8$ and probably would benefit from more resolution. A major problem with the 90-degree case is the ringing or Gibbs phenomenon at the edges.

The original study included tomography performed on real double-exposure holograms as well (ref. 14). A spherical flask immersed in index matching fluid was used to emulate the ball. The pressure within the flask was changed slightly between exposures to create (with difficulty) an approximately uni- 
form change in the index-of-refraction. Heterodyne interferometry (rather than phase shifting interferometry) was used to measure interference phase. In fact, 49 views were each sampled $32 \times 32$ times for a total of 50,176 measurements. The process took about 8 hours. Only 29 views were retained. For this tutorial demonstration, every other measurement was retained and processed by the 10-degree system. Figure 9 compares the results in the central plane for the phantom and the measured data. (The sign depends on which state is chosen as the first exposure.)

The measured result does not have a dip in the center. Surprisingly, the dip appears for the $S=12$ reconstruction, implying that poor performance of the singular value spectrum is not sufficient to reject higher resolutions. Nevertheless, 10-degree viewing cones are probably inadequate for pure tomographic reconstructions.

\section{COMPUTERS AND ARTIFICIAL NEURAL NETWORKS FOR TOMOGRAPHY}

The availability of workstations with large memories makes tomography viable for general flow diagnostics. The software used for the original study mentioned in the previous section was developed on an SGI 4D/25 workstation with 16 megabytes of RAM. However, the $S=12$ case, in the original study, was performed with a supercomputer and about 200 megabytes of RAM. The tutorial demonstration at $S=8$ and with $16 \times 16$ interferograms was performed on an SGI $4 D / 35$ with 128 megabytes of RAM. The results of a single SVD for the tutorial demonstration occupy about 20 megabytes of RAM.

The requirements of the application will determine the computational method and the computer resources needed. The series method probably requires the largest memory. A technique such as ART will require less memory. However, a graphics workstation with at least 96 megabytes of RAM would be a reasonable tool for evaluating computed tomography for an application to flow diagnostics.

Another approach to tomography is to use artificial neural networks to calibrate the procedure (ref. 20). An artificial neural network (fig. 10) is simply a collection of interconnected nonlinear processing elements or nodes. The connections for one node of a feed forward net are shown in figure 10. The classical feed forward net is arranged in layers of nodes. Each layer receives inputs from the previous layer only and sends outputs to the next layer only. The input connections to a node are weighted; the weighted inputs are summed; the sum is passed through a nonlinear function; and the output of the nonlinear function is fanned out to the next layer. A network with at least an input layer, an output layer, and one layer in between (a hidden layer) can be calibrated or trained to perform arbitrary mappings between the input and output layers. The training or calibration procedure consists of using an algorithm to adjust the weights in response to the exemplars in a training set of input-output pairs.

The training sets for the series method are created from phantoms and their calculated interferograms. The phantoms consist of the orthonormal functions and linear combinations. Figure 11 compares the performances of computed tomography and neural net calibration tomography for the 10-degree cone and $S=8$. The neural net had one hidden layer with three nodes. Notation such as e840r is translated

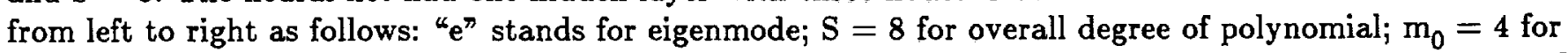
polar index; $\mathrm{m}_{1}=0$ for azimuthal index; and " $\mathrm{r}$ " for real part. The phantom 11 is a linear combination of the first ten azimuthally symmetrical orthonormal functions. The phantom "sphere" is the ball of figures 7 to 9 . Neural net calibration tomography and computed tomography perform similarly.

The times required for SVD and neural-net training or calibration are about the same in software. The potential advantage of neural networks is using dedicated parallel processors that are now becoming 
commercially available. The training and recall speeds of neural net calibration tomography can be many orders of magnitude faster when implemented with the parallel processors.

\section{SOME RESEARCH}

We mentioned that tomography of vector fields is not adequately developed in a mathematical sense. There are high speed measurement methods available in deflectometry that possibly suggest performing such studies.

The exterior problem is solvable with the same general approaches already discussed. Measuring a property distribution exterior to an airfoil is an example of an exterior problem. Evidently, the exterior problem is likely to be more ill-posed than the limited angle problem we have discussed. Some demonstrations would be useful.

The series methods are convenient for combining transforms, model data, and other measurements and for handling incomplete data problems. These methods have demonstrated several defects: they use nonlocal orthonormal functions and require substantial computer resources. The nonlocal problem is being addressed by investigating the use of wavelets for tomography (ref. 21). Wavelets are local functions that can also be used to detect shockwave edges via a method called multiresolution analysis.

Clearly, demonstration applications of different methods of tomography for a variety of flow geometries and properties would be useful.

\section{CONCLUDING REMARKS}

The necessary analytical, detector, and computer technologies are available for tomography as a tool for flow diagnostics. These available technologies do not necessarily mean that tomography is suitable for all applications. It is probably desirable to perform computer experiments using phantom data before committing resources to hardware. Fortunately, small computers are now well equipped for this kind of analysis.

\section{REFERENCES}

1. J. Radon, “Über die Bestimmung von Funktionen durch ihre Integralwerte längs gewisser Mannigfaltigkeiten," Berichte Sächsische Akademie der Wissenschaften, Leipzig, Math.-Phys. Kl. 69, 262-267 (1917).

2. F. Natterer, The Mathematics of Computerized Tomography (Wiley, New York, 1986).

3. S.R. Deans, The Radon Transform and Some of its Applications (Wiley, New York, 1983).

4. A.N. Tikhonov and V.Y. Arsenin, Solutions of Ill-Posed Problems (Winston \& Sons, Washington, 1977).

5. W.H. Press, B.P. Flannery, S.A. Teukolsky, and W.T. Vetterling, Numerical Recipes (Cambridge University Press, New York, 1986). 
6. J. Comput. Assist. Tomogr. (Raven Press, New York).

7. Inverse Probl. (IOP Publishing Ltd, Bristol).

8. R.K. Hanson, J.M. Seitzman, and P.H. Paul, "Planar Laser-Fluorescence Imaging of Combustion Gases," Appl. Phys. B 50, 441-454 (1990).

9. Ref. 2, pp. 92-95.

10. S.H. Izen, "A Series Inversion for the X-ray Transform in n Dimensions," Inverse Probl. 4, 725-748 (1988).

11. S.H. Izen, "Inversion of the k-plane Transform by Orthogonal Function Series Expansions," Inverse Probl. 5, 181-202 (1989).

12. S.H. Izen, "Inversion of the X-ray Transform from Data in a Limited Angular Range," in Signal Processing, Part II: Control Theory and Applications, F.A. Grünbaum, J.W. Helton, and P. Khargonekar, eds. (Springer Verlag, New York, 1990), pp. 275-284.

13. S.H. Izen, An Application for a Limited Solid Angle $X$-ray Transform, Vol. 113 Contemporary Mathematics (American Mathematical Society, Providence, R.I., 1990), pp. 151-170.

14. A.J. Decker and S.H. Izen, "Three-dimensional Computed Tomography from Interferometric Measurements within a Narrow Cone of Views," Appl. Opt. 31, 7696-7706 (1992).

15. Ref. 2, p. 137 .

16. Ref. 2, pp. 137-144.

17. C.M. Vest, Holographic Interferometry (Wiley, New York, 1979), pp. 311-329.

18. R.M. Lewitt, “Reconstruction Algorithms: Transform Methods," IEEE Proc. 71, 390-408 (1983).

19. S.H. Izen, RECON3D Fully Three-Dimensional Tomographic Reconstructions, Manual Version 0.855 (Case Western Reserve University, Cleveland, Oh., 1991).

20. A.J. Decker, "Neural Networks for Calibration Tomography," to be published in SPIE Proc. 2005, Optical Diagnostics in Fluid and Thermal Flow (1993).

21. S.H. Izen, "Frames for the Radon Transform," to be published. 


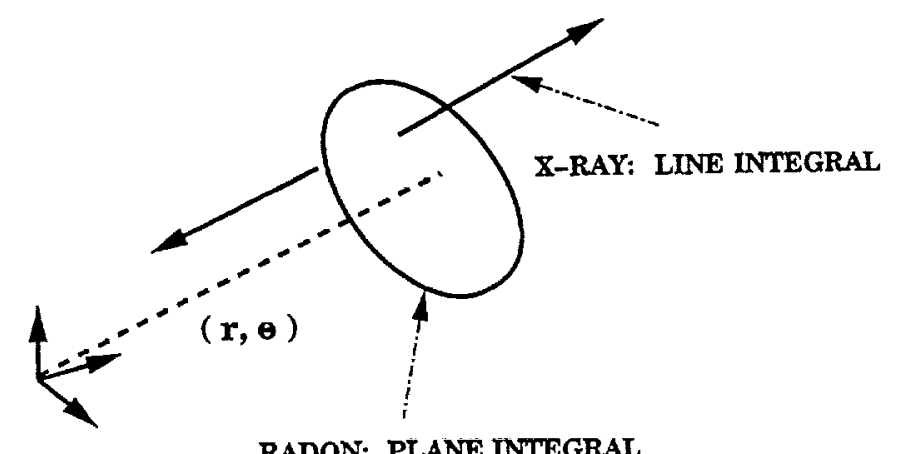

RADON: PLANE INTEGRAL

Figure 1.-The three-dimensional Radon and $\mathrm{x}$-ray transforms.

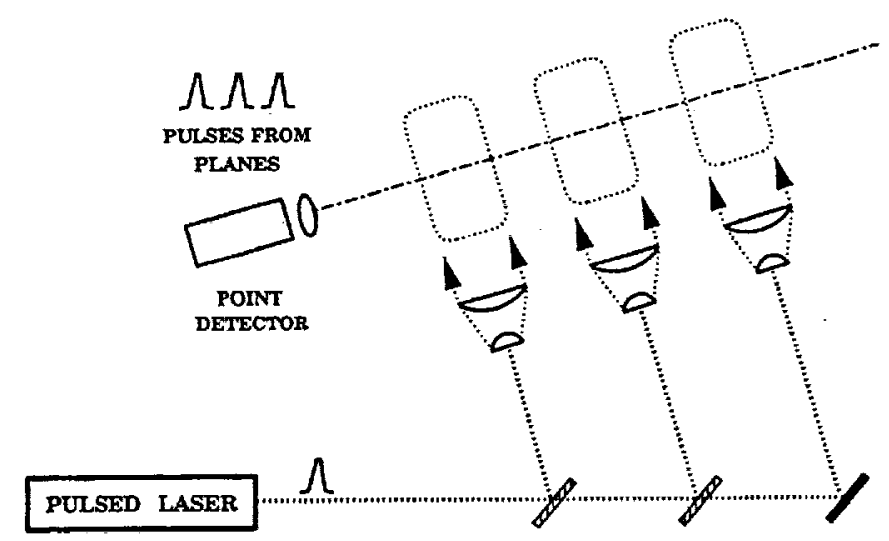

Figure 3.-Measuring three-dimensional Radon transform from sheet fluorescence data. (One direction shown.)

\section{FAST MEASUREMENT OF RADON OR X-RAY TRANSFORM}

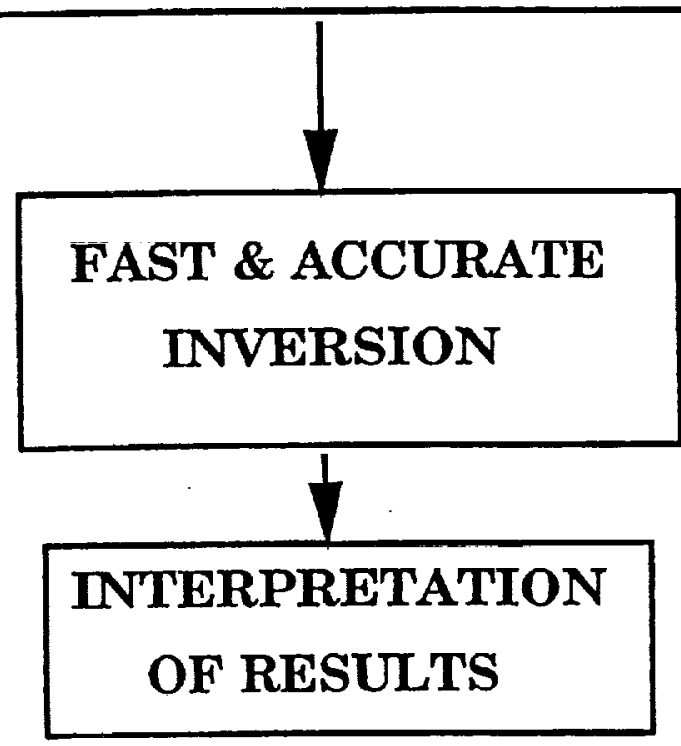

Figure 2.-Organization of tomography into three major subfields.

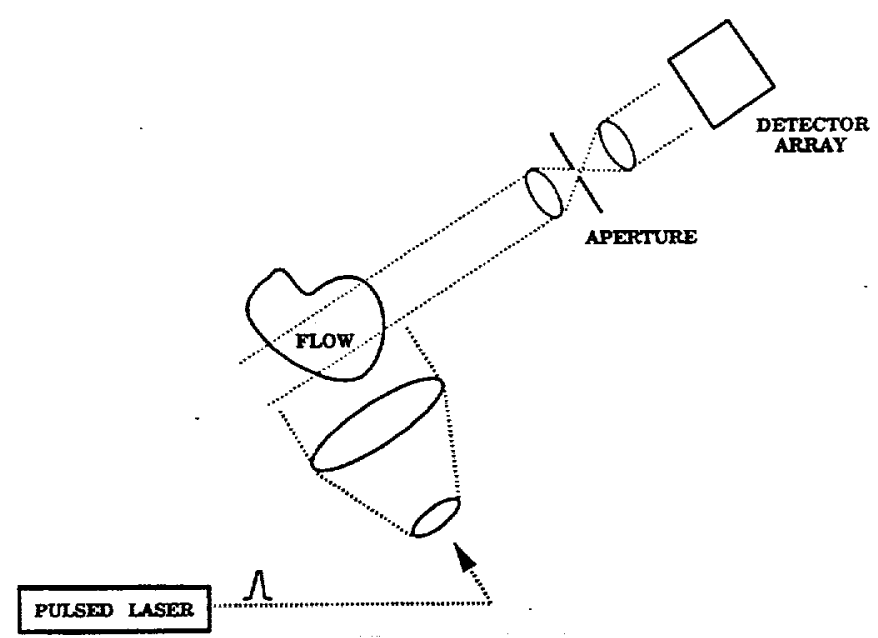

Figure 4.-Measuring three-dimensional $x$-ray transform from fluorescence data. (One direction shown.) 


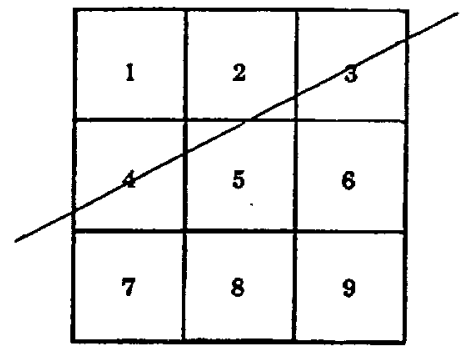

$a_{j 2} f_{2}+a_{j 3} f_{3}+a_{j 4} f_{4}+a_{j 5} f_{5}=g_{j}$

Figure 5-Discretization of two-dimensional slice used in one version of the algebraic reconstruction technique (ART). Each pixel (object element) is weighted by relative length of light ray passing through it.

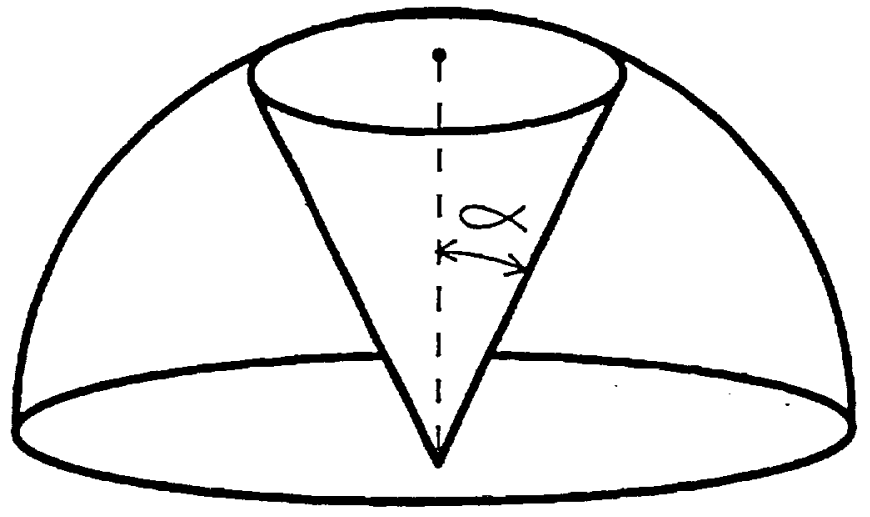

Figure 6.-Limited angle problem in three-dimensional computed tomography ( $\alpha<90$ degrees). Complete data requires sampling directions on entire unit hemispere ( $\alpha=90$ degrees). sing. Value

TEN DEGREE CONE

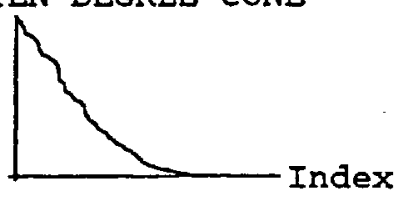

sing. Value

TWO TEN DEGREE CONES

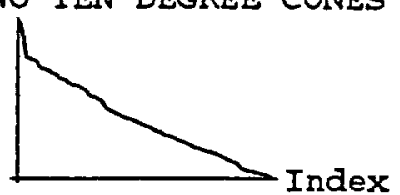

sing. Value

NINTY DEGREE CONE

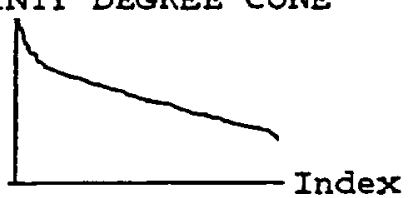

Figure 7.-Relative singular values for the serles method and three viewing situations: one 10-degree cone; two 10-degree cones at right angles; and a full view or 90-degree cone.

Density

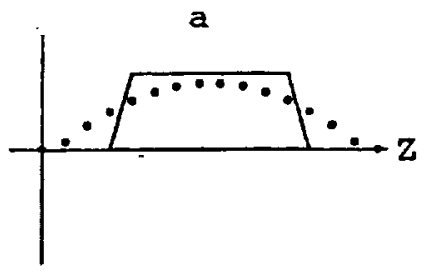

Density

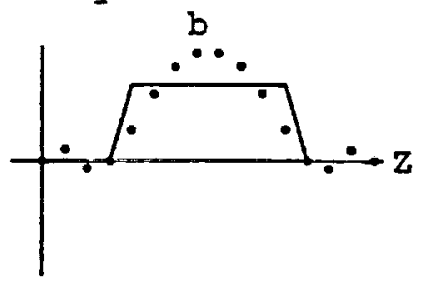

Density

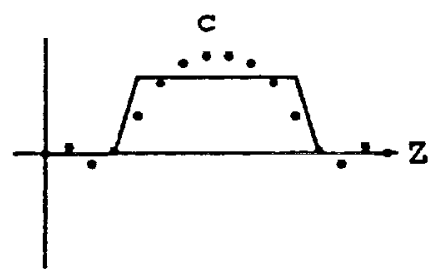

Figure 8.-Reiative densities determined for constant index-of-refraction ball for three viewing situations. (Solid lines represent phantoms and dots represent their reconstructions by computed tomography. Densities are plotted parallel to viewing cone axis.) (a) One 10-degree viewing cone (b) Two 10-degree viewing cones at right angles. (c) Full or 90-degree viewing cone. 

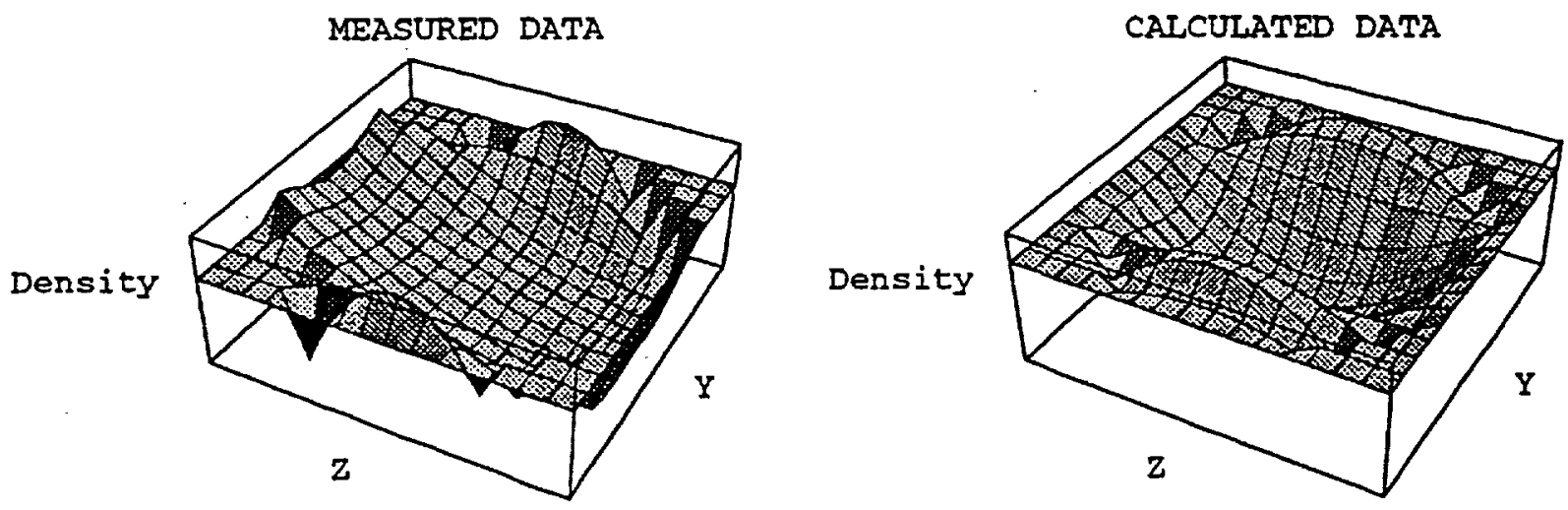

Figure 9.-Comparison of tomography from measured and calculated data for constant index-of-refraction ball for a limited cone angle of 10 degrees; for $2916 \times 16$ interference phase measurements or phantoms; and for a polynomial degree of 8 . (Density is shown near central plane of ball.)

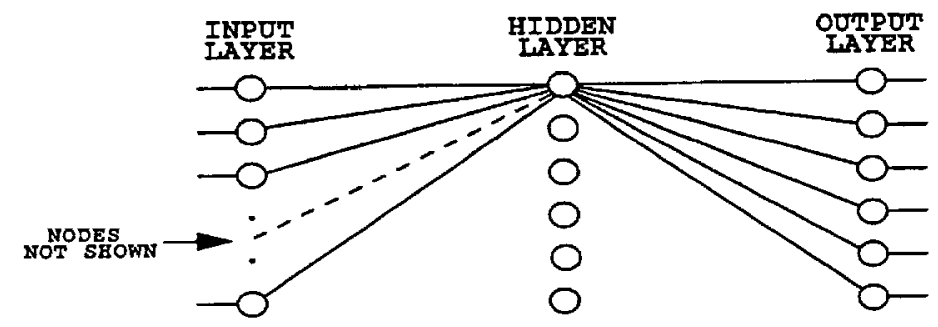

Figure 10.-Sketch of artificial neural network. (Only connections to and from one hidden-layer node are shown.) 


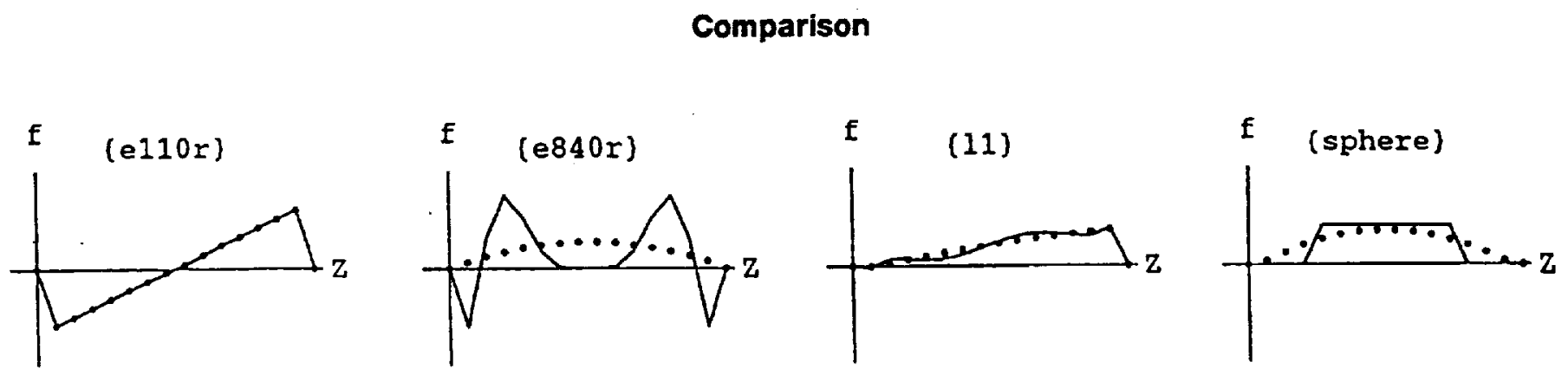

\section{Computed Tomography}
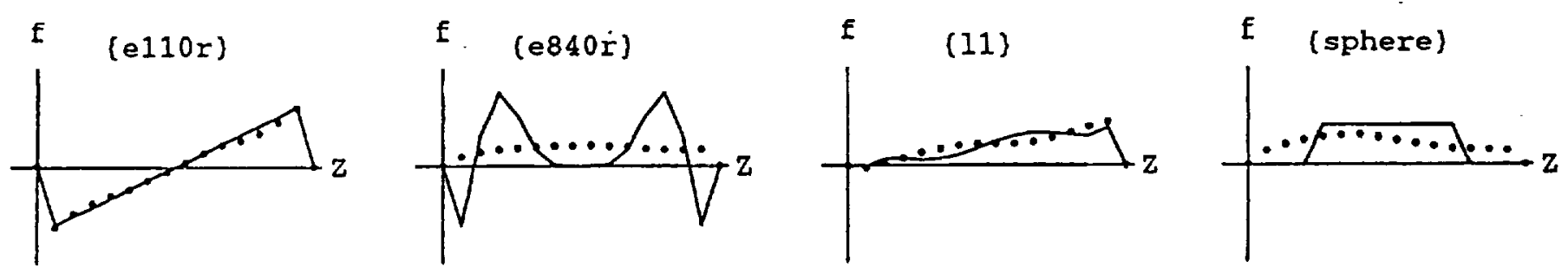

\section{Neural Net Calibration Tomography}

Figure 11. Comparison of performances of computed and neural-net calibration tomography. (Solid lines represent phantoms and dots represent their reconstructions.) 
Public reporting burden for this collection of information is estimated to average 1 hour per response, including the time for reviewing instructions, searching existing data sources, gathering and maintaining the data needed, and completing and reviewing the collection of information. Send comments regarding this burden estimate or any other aspect of this

Davis Highway, Suhe 1204, Arlington, VA 22202-4302, and to the Office of Management and Budget, Paperwork Reduction Project (0704-0188), Washington, DC 20503.

\begin{tabular}{|l|c|c|}
\hline 1. AGENCY USE ONLY (Leave blank) & $\begin{array}{c}\text { 2. REPORT DATE } \\
\text { September } 1993\end{array}$ & $\begin{array}{r}\text { 3. REPORT TYPE AND DATES COVERED } \\
\text { Technical Memorandum }\end{array}$ \\
\hline
\end{tabular}

4. TITLE AND SUBTITLE

Tomographic Methods in Flow Diagnostics
5. FUNDING NUNBERS

WU-505-62-50
6. AUTHOR(S)

Arthur J. Decker
8. PERFORMING ORGANIATION REPORT NUMBER

E-8091

National Aeronautics and Space Administration

Lewis Research Center

Cleveland, Ohio 44135-3191

9. SPONSORING/MONITORING AGENCY NAME(S) AND ADDRESS(ES)

10. SPONSORINGMONITORING AGENCY REPORT NUMBER

National Aeronautics and Space Administration

Washington, D.C. 20546-0001

NASA TM-106330

\section{SUPPLEMENTARY NOTES}

Prepared for the Summer School on Optical Diagnostics for Flow Processes cosponsored by The Danish Research Academy and the European Community Programme on Human Capital and Mobility, Roskilde, Denmark, September 26-October 2, 1993. Responsible person, Arthur J. Decker, (216) 433-3639.

12.. DISTRIBUTION/AVAILABILTV STATEMENT

12b. DISTRIBUTION CODE

Unclassified - Unlimited

Subject Category 35

\section{ABSTRACT (Maximum 200 words)}

This report presents a viewpoint of tomography that should be well adapted to currently available optical measurement technology as well as the needs of computational and experimental fluid dynamists. The goals in mind are to record data with the fastest optical array sensors; process the data with the fastest parallel processing technology available for small computers; and generate results for both experimental and theoretical data. An in-depth example treats interferometric data as it might be recorded in an aeronautics test facility, but the results are applicable whenever fluid properties are to be measured or applied from projections of those properties. The paper discusses both computed and neural net calibration tomography. The report also contains an overview of key definitions and computational methods, key references, computational problems such as ill-posedness, artifacts, and missing data, and some possible and current research topics.

14. SUBJECT TERMS

Tomography; Interferometry; Neural networks; Optical measurements

\begin{tabular}{l|c} 
17. SECURTY CLASSIFICATION \\
$\begin{array}{l}\text { OF REPORT } \\
\text { Unclassified }\end{array}$
\end{tabular} $\begin{gathered}\text { 18. SECURTY CLASSIFICATION } \\
\text { OF THIS PAGE } \\
\text { Unclassified }\end{gathered}$

19. SECUATY CLASSIFICATION OF ABSTRACT 